\title{
On the domain of validity of the near-parallel combined stability analysis for the 2D intermediate and far bluff body wake
}

\author{
Daniela Tordella $^{1, *}$ and Marco Belan ${ }^{2}$ \\ ${ }^{1}$ Politecnico di Torino, Dept. of Aerospace Engineering, 10129 Torino, Italy \\ ${ }^{2}$ Politecnico di Milano, Dept. of Aerospace Engineering, Campus Bovisa, Milano, Italy
}

Received 4 April 2003, accepted 18 March 2004

Published online 10 January 2005

Key words nonparallel instability, asymptotic expansion, laminar, wake basic flows. MSC (2000) 76D $25,76 \mathrm{M} 45$

Local parallelism is a representation scheme of flow instability, which is founded on the assumption of local uniformity of the flow in the streamwise direction, the global disuniformity being given by the slow variations of the local values of the equilibrium flow solution. Since the remarkable numerical experiment by Triantafyllou and Karniadakis [46] proved that the details of the flow separation from the body generating the wake can be disregarded in wake-stability analyses and since the intermediate and far regions of the basic wake flow are actually physical systems which show a slow streamwise evolution, it is here considered - as a first level of extension of the near-parallel analysis - the hypothesis that this evolution influences the instability characteristics mainly through a slow spatial modulation of the instability wave. To determine the domain of validity of the assumption of local parallellism for the two dimensional bluff body wake, a non local analysis of the combined stability of the intermediate and far field regions of the flow has been carried out using the method of multiple spatial scales. The analysis takes into account nonparallel flow effects, such as the streamwise variations of the mean flow, of the disturbance profile function and its longitudinal modulation and of the wavenumber and spatial growth rate. The nonparallel effects are introduced through a basic field - a Navier-Stokes expansion solution that represents the intermediate and far regions of the wake - which involves the streamwise and transverse momentum components as functions of both the coordinates. After the introduction of the slow streamwise variable, this leads to a zero order parametric Orr-Sommerfeld eigenvalue problem and to a first order nonparallel correction, which is represented by a non homogeneous Orr-Sommerfeld equation. The first order correction results in remarkable variations of perturbation wave numbers and spatial growth rates. These variations are distributed in the intermediate region of the field, their intensity increase getting closer to the body up to a level which yields the break-up of the near-parallellism assumption. The break-up point is placed well downstream to the region occupied by the standing vortices. The theory allows one to determine the distance from the body beyond which the local parallel analysis is fully valid. The continuous spectrum of the parametric Orr-Sommerfeld combined eigenvalue problem is also determined. It has been shown that, according to the Briggs criterium, the continuum cannot give rise to unstable single modes.

\section{Introduction}

Over the past thirty years it has been usual to study the stability of two dimensional incompressible wakes by locally neglecting the divergence of the steady primary flow. The local quasi-parallel assumption was the salient feature of most of the linear stability analysis that have appeared in literature, (e.g. Mattingly and Criminale [26], Koch [24], Triantafyllou et al. [45], Triantafyllou et al. [44], Hultgren and Aggarwal [21], Hannemann and Oertel [19]), which presume either a two dimensional perturbation (modal approach) or a pulsewise (in space and time) perturbation and which were applied to velocity profiles of either experimental or numerical origin.

All these stability analyses identified the region where the instability originates - a pocket of absolute instability of finite extension followed by a region of convective instability - in the very near part of the wake, that is, whereabouts the streamlines are far from being parallel and show the maximum curvature of the wake field. This region leads to global linear instability whenever the critical value of the flow control parameter is exceeded. Details change according to the frequency selection criteria that are adopted (see, for example, Koch [24], Pierrehumbert [35], Monkewitz and Nguyen [28]. All these criteria rely on the concept of local absolute and convective unstable wave propagation, that historically originates from the mathematical model used to describe the instabilities of plasma flows, see Twiss [49], Sturrock [42], Briggs [10], Bers [8],

\footnotetext{
* Corresponding author, e-mail: daniela.tordella@ polito.it
} 
and which, more recently, has very often been applied to hydrodynamics stability (see the reviews by Oertel [34] and Huerre and Monkewitz [20]).

It should be recalled, however, that, on the basis of a combined inviscid stability analysis coupled to an experimental investigation of naturally amplifying disturbances, a different interpretation, in terms of only the spatial viewpoint, was given by Mattingly and Criminale [26] of the downstream development of the wave characteristics of a thin airfoil wake. It is also necessary to recall the numerical experiments by Jackson [22], Zebib [53] and Morzinski and Thiele [29, 30], where the eigenvalues of the whole 2D flow were calculated, the 3D numerical global stability analysis of the steady and periodic wake by Noak and Eckelmann [32] and the laboratory experiment on the temporally growing disturbances in near-critical and super-critical wakes by Provansal et al. [36]. Intensive experimental laboratoty research was also dedicated to the vortex shedding sensitivity to perturbations in the near-wake region, see e.g. Roshko [37], Tritton [47,48], Berger [4], Berger and Wille [5], Gaster [15, 16], Williamson [51], Strykowsky and Sreenivasan [41] and Roussopoulos [38]. Furthermore, a direct numerical simulation by Triantafyllou and Karniadakis [46] showed that the vortex wake can be reproduced by only knowing the mean velocity profile at the station behind the object which is the most unstable according to the near-parallel linear instability theory.

In this paper the authors consider the questions: Is the local parallelism - an assumption which requires, in short, that the properties of the wake are adequately represented, locally, at each section behind the body, by the stability properties of a parallel flow with the same average velocity profile - such a restrictive assumption, whose partial realease, through a spatial multiscaling expansion truncated at the first order term, could remarkably change the stability characteristics? At what distance from the body do the quasi-parallel and the nonparallel theories coalesce?

On the other hand, the local assumption of parallellism, as well as the hypothesis of linearity, cannot irremediably compromise the physical correctness of the phenomenon representation, but can only limit their domain of validity. As usually happens when working with both the linear and near-equilibrium formalism, it is not completely a priori clear which is the actual domain of validity. However, a domain of validity must exist, for small enough values of the streamline curvature. This domain would surely include the far wake, which is asymptotically a parallel flow, but it could also include as well a large part of the intermediate wake.

In this article, the term intermediate is used in the general sense as used by Zeldovich and Barenblatt (e.g. G.I. Barenblatt [2]: ' . . intermediate asymptotics are self-similar or near-similar solutions of general problems, valid for times, and distances from boundaries, large enough for the influence of the fine details of the initial/or boundary conditions to disappear, but small enough that the system is far from the ultimate equilibrium state...' (ib., Preface, page Xiii)).

Since the basic (intermediate and far) flow is actually a system which shows a slow spatial evolution, it is here considered - as a first level of extension of the near-parallel analysis presented in previous literature - the hypothesis that this evolution influences the instability characteristics mainly through a slow spatial modulation of the instability wave. Under the frame of a spatial multiscale analysis, such a modulation may thus be represented in terms of a correction acting on the complex wave number. According to this analysis, in the regions of the wake where the correction turns out to be small, the near parallelism is confirmed, in the regions where the correction is large, the opposite holds.

In this article, the authors follow Nayfeh [31] and Saric and Nayfeh [40], and present a nonparallel combined stability theory for two dimensional incompressible wakes (Sects. 2, 2.2, 2.3). The domain of the present investigation is a portion of the wake, but the near wake which is highly nonparallel, where a criterium of near-parallelism such as

$$
\epsilon \sim \frac{\lambda}{L} \ll 1
$$

may be considered fullfilled ( $\lambda$ being the typical instability wave length and $L$ the slow evolution length scale of the flow). Assuming $L \sim R \lambda$ yields the small dimensionless parameter $\varepsilon$, which characterizes the nonparallelism of the primary flow, and thus the scale of the slow streamwise variation, the value $\varepsilon \sim 1 / R$. The multiple scales method, as here applied, relies on the introduction of this paramenter, which characterizes the decomposition of the nonparallel primary flow. The primary flow is a Navier-Stokes asymptotic expansion in terms of the inverse coordinates, which accounts for the effects of the streamwise diffusion and non linear convection, and entrainment from the sides (see Sect. 2.1 and Belan and Tordella, 2002 [3]; Tordella and Belan, 2003 [43]). The spatial multiscaling also relies on an expanded perturbative equation, which is here truncated at the first order of accuracy with regards to $\epsilon$. The continuous spectrum associated to the parametric Orr-Sommerfeld problem is derived in Sect. 3 and the role played by the continuum on the stability of the wake is discussed.

The theory is applied to the wake of the circular cylinder (Sect. 4). Finally, conclusions are given in Sect. 5. 


\section{Problem formulation and method of solution}

The stability of a two-dimensional, steady, incompressible flow past a bluff body, which is described by the stream function $\Psi(x, y)$, is considered. The $x$ coordinate is parallel to the free stream velocity, while the $y$ coordinate is normal. Dimensionless quantities, normalized using the body reference length $D$ and the free stream velocity $U$ (so that $R=U D / \nu$ ), are introduced.

The here considered stream function $\Psi(x, y)$ is that of the intermediate and far portions of the bluff-body wake described by an asymptotic expansion obtained from the matching of an inner Navier-Stokes thin shear flow solution and an outer Navier-Stokes solution (Belan and Tordella, 2002 [3]; Tordella and Belan, 2003 [43]). Details about this asymptotic NavierStokes expansion are given in Sect. 2.1.

To examine the stability of such a flow, small disturbances are superposed on it. The stream function of the disturbed flow is considered to have the form $\Psi(x, y)+\psi(x, y, t)$. Substituting this disturbed stream function in the Navier-Stokes equations, subtracting the mean flow quantities and keeping the linear terms in $\psi$, yields the linearized perturbation equation

$$
\partial_{t} \nabla^{2} \psi+\left(\partial_{x} \nabla^{2} \Psi\right) \psi_{y}+\Psi_{y} \partial_{x} \nabla^{2} \psi-\left(\partial_{y} \nabla^{2} \Psi\right) \psi_{x}-\Psi_{x} \partial_{y} \nabla^{2} \psi=\frac{1}{R} \nabla^{4} \psi,
$$

whose appropriate boundary conditions along the $y$ coordinate are

$$
\begin{array}{r}
\lim _{|y| \rightarrow \infty} \psi(x, y)=0, \\
\lim _{|y| \rightarrow \infty} \partial_{y} \psi(x, y)=0 .
\end{array}
$$

The scale

$$
x_{1}=\varepsilon x
$$

is used to express the slow variation of the basic field along the $x$ coordinate, where $\varepsilon$ is the small dimensionless parameter that characterises the nonparallelism of the primary flow and which is assumed (see the introduction) to be given by $\varepsilon=1 / R$ (according to a thin shear layer hypotesis assumption for wake flows with $R$ inside the $[30,100]$ interval). Thus, the basic flow hypothesis $\Psi=\Psi\left(x_{1}, y\right)$ is made. In this way the basic (intermediate and far) flow is actually a system which shows a slow streamwise evolution. This hypothesis is here accompanied, as a first step towards an extension of the near-parallel analysis, by the hypothesis that this evolution influences the instability characteristics mainly through a slow spatial modulation of the instability wave, which, under the frame of a spatial multiscale analysis, can be represented in terms of a correction acting on the complex wave number (see Sects. 2.2 and 2.3). According to this analysis, in the regions of the wake where the correction turns out to be small, the near parallelism is confirmed, in the regions where the correction is large, the opposite holds.

The following perturbation hypothesis is therefore introduced (Nayfeh [31] and Saric and Nayfeh [40])

$$
\psi=\varphi\left(x_{1}, y ; \varepsilon\right) \mathrm{e}^{i \theta(x, t ; \varepsilon)}=\left[\varphi_{0}\left(x_{1}, y\right)+\varepsilon \varphi_{1}\left(x_{1}, y\right)+\ldots\right] \mathrm{e}^{i \theta(x, t ; \varepsilon)} .
$$

According to the Whitham theory [50],

$$
\begin{aligned}
& \partial_{x} \theta=h_{0}=k_{0}+\mathrm{i} s_{0}, \\
& \partial_{t} \theta=-\sigma=-(\omega+\mathrm{i} r),
\end{aligned}
$$

where $h_{0}$ is the complex dimensionless wave number and $\sigma$ is the complex dimensionless frequency. The imaginary parts of $h_{0}$ and $\sigma$ are the spatial and temporal growth rates, respectively. In terms of $x_{1}$ and $\theta$ the spatial and temporal derivatives transform, according to the change in variables $\{x, y, t\} \rightarrow\left\{x_{1}, y, \theta\right\}$, as

$$
\begin{aligned}
\frac{\partial}{\partial x} & =h_{0} \frac{\partial}{\partial \theta}+\varepsilon \frac{\partial}{\partial x_{1}}, \\
\frac{\partial}{\partial y} & =\frac{\partial}{\partial y}, \\
\frac{\partial}{\partial t} & =-\sigma \frac{\partial}{\partial \theta} .
\end{aligned}
$$

Eventually, by applying this transformation to perturbation eq. (2), an expanded perturbation equation, which is equivalent to the following sequence of perturbative equations, is obtained: 
$-\varepsilon^{0}$ (homogeneous ODE for $\varphi_{0}$, depending on the $y$ coordinate, where $x_{1}$ and $R$ are parameters), $-\varepsilon^{1}$ (non homogeneous ODE for $\varphi_{1}$, depending on the $y$ coordinate, where $x_{1}$ and $R$ are parameters, the n.h.t. depending on $x_{1}, y, \varphi_{0}, h, \sigma$ and $R),-O\left(\varepsilon^{2}\right)$.

The zero order equation is the parametric Orr-Sommerfeld equation, which gives the basic approximation of the field dispersion relationship. From the non homogeneous equation of $O(\varepsilon)$ it is possible to derive the condition which allows one to determine the nonparallel corrections on the complex wave number (see Sect. 2.3).

\subsection{Nonparallel basic flow}

An approximated Navier-Stokes solution for the region of the two dimensional steady bluff body wake where the nonparallelism of the streamlines is not yet negligible has been considered. This region is intermediate between the field on the body scale $L_{e}$, which includes the two symmetrical counter rotating eddies, and the ultimate far wake. The analytical representation here used accounts for the effects of the streamwise diffusion, non linear convection and entrainment at the lower orders and for the pressure gradient and linear and non linear exchange of vorticity from the inner toward the outer part of the flow at the higher orders (Belan and Tordella [3]; Tordella and Belan [43]). At the first order in $\epsilon$, the multiscaling, as applied to such a basic field, allows the insertion of the above mentioned lower order effects, through, perhaps for the first time, the explicit use of the transversal momentum component of the primary flow. The solution was obtained by matching an inner solution - a Navier-Stokes expansion in powers of the inverse of the longitudinal coordinate $\left(x^{-n / 2}, n=0,1,2, \ldots\right)$ - and an outer solution, which is a Navier-Stokes asymptotic expansion in powers of the inverse of the distance from the body. The matching was built on the criteria that, where the two solutions meet, the pressure longitudinal gradients and the vorticities must be equal and the flow towards the inner layer must be equal to the outflow from the external stream. The lateral decay results to be algebraic at high orders in the inner expansion solution. Assuming the inner expansion as being an approximation of the primary wake flow, using the quasi-similar transformation

$$
x=x, \eta=x^{-1 / 2} y
$$

the velocity components, up to $O\left(x^{-3 / 2}\right)$, can be written as

$$
\begin{aligned}
& u=1+x^{-1 / 2} \phi_{1}(\eta)+x^{-1} \phi_{2}(\eta)+x^{-3 / 2} \phi_{3}(\eta), \\
& v=x^{-1} \chi_{1}(\eta)+x^{-3 / 2} \chi_{2}(\eta) .
\end{aligned}
$$

According to the multiscale approach, see (5), (6), $\eta=\left(R x_{1}\right)^{-1 / 2} y$ and the velocity components of the basic flow can also be written as

$$
\begin{aligned}
& u=\partial_{y} \Psi=u_{0}\left(x_{1}, y\right)+\varepsilon u_{1}\left(x_{1}, y\right)+\ldots, \\
& v=-\partial_{x} \Psi=-\varepsilon \partial_{x_{1}} \Psi=\varepsilon v_{1}\left(x_{1}, y\right)+\ldots .
\end{aligned}
$$

By only considering the integer powers of $\varepsilon$, eqs. (13), (14) can assume the multiscale structure (15), (16). For the $u$ component it results that

$$
\begin{aligned}
u & =1+R^{-1 / 2} x_{1}^{-1 / 2} \phi_{1}\left(y / \sqrt{R x_{1}}\right)+R^{-1} x_{1}^{-1} \phi_{2}\left(y / \sqrt{R x_{1}}\right)+R^{-3 / 2} x_{1}^{-3 / 2} \phi_{3}\left(y / \sqrt{R x_{1}}\right) \\
& =\left[1+R^{-1 / 2} x_{1}^{-1 / 2} \phi_{1}(\eta)\right]+\varepsilon\left[x_{1}^{-1} \phi_{2}(\eta)+R^{-1 / 2} x_{1}^{-3 / 2} \phi_{3}(\eta)\right],
\end{aligned}
$$

so that

$$
\begin{aligned}
u_{0}= & 1+R^{-1 / 2} x_{1}^{-1 / 2} \phi_{1}=1-x_{1}^{-1 / 2} R^{-1 / 2} a \mathrm{e}^{-\left(R \eta^{2}\right) / 4}, \\
u_{1}= & x_{1}^{-1} \phi_{2}+R^{-1 / 2} x_{1}^{-3 / 2} \phi_{3} \\
= & -x_{1}^{-1} \frac{A^{2}}{2} \mathrm{e}^{-\frac{R}{4} \eta^{2}}\left\{b_{1} \mathrm{~F}_{1}\left(-\frac{1}{2}, \frac{1}{2} ; \frac{R}{4} \eta^{2}\right)+\mathrm{e}^{-\frac{R}{4} \eta^{2}}+\frac{\sqrt{\pi R}}{2} \eta \operatorname{erf}\left(\frac{\sqrt{R}}{2} \eta\right)\right\} \\
& -x_{1}^{-3 / 2} R^{-1 / 2} a^{3}\left(R \eta^{2}-2\right)\left[c-R \mathrm{H}_{\mathrm{r} 2}(\eta) F_{3}(\eta)\right],
\end{aligned}
$$

where constants $a, b$, and $c$ are integration constants that depend on the Reynolds number (see Belan and Tordella [3]). For the $[30,60] R$ range the constants have been interpolated by using the experimental data by Kovásznay [25] and the numerical data by Berrone [6,7]; for the [60,100] range by using the numerical data by Dennis and Chang [13], Fornberg [14] and Berrone [6,7]. Function ${ }_{1} \mathrm{~F}_{1}$ is the confluent hypergeometric function (see Kamke [23]), $\mathrm{H}_{\mathrm{r} 2}(\eta)=\mathrm{H}_{2}\left(\frac{1}{2} R^{1 / 2} \eta\right)$ is 
the particular function, for $n=3$, of the general $n t h$-order function $\mathrm{H}_{\mathrm{r} n-1}(\eta)=\mathrm{H}_{n-1}\left(\frac{1}{2} R^{1 / 2} \eta\right)\left(\mathrm{H}_{n}\right.$ are Hermite's polynomials), and $F_{3}$ is the particular case, for $n=3$, of the general $n t h$-order function

$$
\begin{aligned}
& F_{n}(\eta)=\int \frac{\mathrm{e}^{\frac{R}{4} \eta^{2}}}{\mathrm{H}_{\mathrm{r}_{n-1}}^{2}(\eta)} G_{n}(\eta) \mathrm{d} \eta \\
& G_{n}(\eta)=a^{-n} \int M_{n}(\eta) \mathrm{H}_{\mathrm{r} n-1}(\eta) \mathrm{d} \eta .
\end{aligned}
$$

Function $M_{n}(\eta)$ is the sum of the non homogeneous terms of the general ordinary differential equation for $\phi_{n}, n \geq 1$, obtained from the $x$ component of the Navier-Stokes equation (see Tordella and Belan [43]). Function $M_{n}$ includes the effects of the streamwise pressure gradient and diffusion terms and also of part of the longitudinal convection term.

At the same approximation order, the $v$ component is given by

$$
\begin{aligned}
v & =R^{-1} x_{1}^{-1} \chi_{1}\left(x_{1}, y\right)+R^{-3 / 2} x_{1}^{-3 / 2} \chi_{2}\left(x_{1}, y\right) \\
& =\varepsilon\left[x_{1}^{-1} \chi_{1}(\eta)+R^{-1 / 2} x_{1}^{-3 / 2} \chi_{2}(\eta)\right]
\end{aligned}
$$

so that

$$
\begin{aligned}
v_{1} & =x_{1}^{-1} \chi_{1}+x_{1}^{-3 / 2} R^{-1 / 2} \chi_{2} \\
& =\frac{1}{2} x_{1}^{-3 / 2} R^{-1 / 2}\left\{-a\left(R x_{1}\right)^{1 / 2} \eta \mathrm{e}^{-\left(R \eta^{2}\right) / 4}+\left[\Phi_{2}(\eta)+\frac{y}{\sqrt{R x_{1}}} \phi_{2}(\eta)\right]\right\}
\end{aligned}
$$

where $\phi_{2}$ is the function coefficient of the $u$ expansion at the order $x^{-1}$, which is also present in (18), and $\Phi_{2}=\int \phi_{2} \mathrm{~d} \eta$.

\subsection{Zero order theory}

Substituting eqs. (2.5)-(2.7), (2.8)-(2.10) in eqs. (2.1)-(2.3) and using eqs. (2.14)-(2.15), at the order $\varepsilon^{0}$, the eigenvalue equation (parametric Orr-Sommerfeld) is obtained

$$
\mathcal{A} \varphi_{0}=\sigma \mathcal{B} \varphi_{0}
$$

where $\mathcal{A}$ and $\mathcal{B}$ are the standard Orr-Sommerfeld linear differential operators

$$
\begin{aligned}
& \mathcal{A}=\left\{\left(\partial_{y}^{2}-h_{0}^{2}\right)^{2}-\mathrm{i} h_{0} R\left[u_{0}\left(\partial_{y}^{2}-h_{0}^{2}\right)-u_{0}^{\prime \prime}\right]\right\}, \\
& \mathcal{B}=-\mathrm{i} R\left(\partial_{y}^{2}-h_{0}^{2}\right),
\end{aligned}
$$

except that $\mathcal{A}$ depends on $x_{1}$ through $u_{0}$, so that $x_{1}$ is a parameter of the whole equation. After having considered

$$
h_{0} \text { as parameter } \Longrightarrow \sigma \text { as eigenvalue, }
$$

both the eigenvalues $\sigma\left(x_{1} ; h_{0}, R\right)$ and eigenfunctions $\varphi_{0}\left(x_{1}, y ; h_{0}, R\right)$ also have a local meaning.

The boundary conditions for proper eigenfunctions are

$$
\begin{aligned}
\varphi_{0} & \rightarrow 0 \text { as }|y| \rightarrow \infty, \\
\partial_{y} \varphi_{0} & \rightarrow 0 \text { as }|y| \rightarrow \infty .
\end{aligned}
$$

The solution $\varphi_{0} \in L^{2}(-\infty, \infty)$, it has a finite norm $\left\|\varphi_{0}\right\|_{L^{2}}=\left(\varphi_{0}, \varphi_{0}\right)<\infty$. Since the Orr-Sommerfeld equation is not self-adjoint and the present domain is unbounded, it is not certain that a complete discrete spectrum of eigenvalues exists. It is thus necessary to directly check the instability properties of both the discrete and continuous spectra (see Sect. 3).

In order to solve eq. (21) in the multiscaling frame, it is useful to write

$$
\varphi_{0}\left(x_{1}, y\right)=A\left(x_{1}\right) \zeta_{0}\left(x_{1}, y\right)
$$

so that eqs. (21), (22) and (23) become

$$
\begin{aligned}
& \mathcal{A} \zeta_{0}=\sigma \mathcal{B} \zeta_{0}, \\
& \quad \zeta_{0} \rightarrow 0 \text { as }|y| \rightarrow \infty,
\end{aligned}
$$




$$
\partial_{y} \zeta_{0} \rightarrow 0 \text { as }|y| \rightarrow \infty
$$

Function $A\left(x_{1}\right)$, the slow modulation, is determined at the next order of approximation. The system (25)-(27) can be solved by standard numerical techniques, giving, apart from a proportionality coefficient, the eigenfunction $\zeta_{0}$ and the discrete set of eigenvalues $\left\{\sigma_{n}\left(x_{1} ; h_{0}, R\right)\right\}$. By selecting the eigenvalue with the largest imaginary part (simply denoted as $\sigma$ in what follows), a first approximation of the dispersion relation $D\left(x_{1} ; h_{0}, \sigma, R\right)=0$ is obtained in the explicit form

$$
\sigma=\sigma\left(x_{1} ; h_{0}, R\right) \text {. }
$$

A further analysis of this relation gives, according to the Briggs criterion [10] the loci of the branching points $\left\{h_{0 s}\left(x_{1}\right), \sigma_{s}\left(x_{1}\right)\right\}$ in the complex Fourier space where

$$
\frac{\partial \sigma}{\partial h_{0}}=0
$$

(see also Huerre and Monkewitz [20]). This leads to a zero order estimate of the local instability typology, which is absolute if $r_{s}>0$, and convective otherwise.

\subsection{First order theory}

The resulting equation at $O(\epsilon)$ is a non homogeneous Orr-Sommerfeld equation

$$
\mathcal{A} \varphi_{1}=\sigma \mathcal{B} \varphi_{1}+\mathcal{M} \varphi_{0}
$$

where $\mathcal{M}$ is the linear differential operator

$$
\begin{aligned}
\mathcal{M}= & \left\{\left[R\left(2 h_{0} \sigma-3 h_{0}^{2} u_{0}-u_{0}^{\prime \prime}\right)+4 \mathrm{i} h_{0}^{3}\right] \partial_{x_{1}}+\left(R u_{0}-4 \mathrm{i} h_{0}\right) \partial_{x_{1} y y}^{3}\right. \\
& \left.+R v_{1}\left(\partial_{y}^{3}-h_{0}^{2} \partial_{y}\right)-R v_{1}^{\prime \prime} \partial_{y}+\mathrm{i} h_{0} R\left[u_{1}\left(\partial_{y}^{2}-h_{0}^{2}\right)-u_{1}^{\prime \prime}\right]\right\} .
\end{aligned}
$$

The relevant boundary conditions for the discrete part of the spectrum are

$$
\begin{aligned}
\varphi_{1} & \rightarrow 0 \text { as }|y| \rightarrow \infty, \\
\partial_{y} \varphi_{1} \rightarrow 0 & \text { as }|y| \rightarrow \infty .
\end{aligned}
$$

Eq. (30) can now be used to obtain the modulation function $A\left(x_{1}\right)$, which was left undetermined at the zero level of approximation, and to obtain the first order corrections to the loci of the previously determined branching points. Let us write, by means of (24), $\mathcal{M} \varphi_{0}$ in the form $\mathcal{M} A \zeta_{0}$, which can be rearranged as:

$$
\begin{aligned}
\mathcal{M} A \zeta_{0} & =\mathrm{d}_{x_{1}} A\left\{\left[F_{1}+F_{2} \partial_{y}^{2}\right]+A\left[F_{1} \partial_{x_{1}}+F_{2} \partial_{x_{1} y y}^{3}+F_{3}+F_{4} \partial_{y}+F_{5} \partial_{y}^{2}+F_{6} \partial_{y}^{3}\right]\right\} \zeta_{0} \\
& =\hat{\mathcal{M}} \zeta_{0}
\end{aligned}
$$

where the $F$ 's are known functions of $x_{1}, y$

$$
\begin{aligned}
& F_{1}=R\left(2 h_{0} \sigma-3 h_{0}^{2} u_{0}-u_{0}^{\prime \prime}\right)+4 \mathrm{i} h_{0}^{3}, \\
& F_{2}=R u_{0}-4 \mathrm{i} h_{0}, \\
& F_{3}=-\mathrm{i} h_{0} R\left(\partial_{y}^{2}-h_{0}^{2}\right) u_{1}, \\
& F_{4}=-R\left(\partial_{y}^{2}-h_{0}^{2}\right) v_{1}, \\
& F_{5}=\mathrm{i} h_{0} R u_{1} \\
& F_{6}=R v_{1} .
\end{aligned}
$$

Eq. (30) has a solution if, and only if, the non homogeneous term is orthogonal to every solution of the adjoint homogeneous problem. This problem can be written, by considering the hermitian conjugate equation,

$$
\tilde{\mathcal{A}} \tilde{\zeta}_{0}=\sigma^{*} \tilde{\mathcal{B}} \tilde{\zeta}_{0}
$$


where $*$ denotes the complex conjugation, and

$$
\begin{aligned}
& \tilde{\mathcal{A}}=\left\{\left(\partial_{y}^{2}-h_{0}^{* 2}\right)^{2}-\mathrm{i} h_{0}^{*} R\left[\left(\partial_{y}^{2}-h_{0}^{* 2}\right) u_{0}-u_{0}^{\prime \prime}\right]\right\}, \\
& \tilde{\mathcal{B}}=\mathrm{i} R\left(\partial_{y}^{2}-h_{0}^{* 2}\right) .
\end{aligned}
$$

The same equation can be rewritten in the complex-conjugate form

$$
\mathcal{A}^{+} \zeta_{0}^{+}=\sigma \mathcal{B} \zeta_{0}^{+}
$$

where

$$
\mathcal{A}^{+}=\left\{\left(\partial_{y}^{2}-h_{0}^{2}\right)^{2}-\mathrm{i} h_{0} R\left[\left(\partial_{y}^{2}-h_{0}^{2}\right) u_{0}-u_{0}^{\prime \prime}\right]\right\}
$$

and $\mathcal{B}=\tilde{\mathcal{B}}^{*}$. Now, the orthogonality condition is

$$
\left(\tilde{\zeta}_{0}, \hat{\mathcal{M}} \zeta_{0}\right) \equiv \int_{-\infty}^{\infty} \zeta_{0}^{+} \hat{\mathcal{M}} \zeta_{0} \mathrm{~d} y=\int_{-\infty}^{\infty} \zeta_{0}^{+} \mathcal{M} A \zeta_{0} \mathrm{~d} y=0
$$

Using the explicit form of $\mathcal{M}$, this condition becomes

$$
\left(\mathrm{d}_{x_{1}} A\right) \int_{-\infty}^{\infty} \zeta_{0}^{+}\left[F_{1}+F_{2} \partial_{y}^{2}\right] \zeta_{0} \mathrm{~d} y+A \int_{-\infty}^{\infty} \zeta_{0}^{+}\left[F_{1} \partial_{x_{1}}+F_{2} \partial_{x_{1} y y}^{3}+F_{3}+F_{4} \partial_{y}+F_{5} \partial_{y}^{2}+F_{6} \partial_{y}^{3}\right] \zeta_{0} \mathrm{~d} y=0
$$

or, in short form,

$$
I_{1}\left(x_{1}\right) \mathrm{d}_{x_{1}} A\left(x_{1}\right)+I_{2}\left(x_{1}\right) A\left(x_{1}\right)=0,
$$

where

$$
\begin{aligned}
& I_{1}\left(x_{1}\right)=\int_{-\infty}^{\infty} \zeta_{0}^{+}\left[F_{1}+F_{2} \partial_{y}^{2}\right] \zeta_{0} \mathrm{~d} y \\
& I_{2}\left(x_{1}\right)=\int_{-\infty}^{\infty} \zeta_{0}^{+}\left[F_{1} \partial_{x_{1}}+F_{2} \partial_{x_{1} y y}^{3}+F_{3}+F_{4} \partial_{y}+F_{5} \partial_{y}^{2}+F_{6} \partial_{y}^{3}\right] \zeta_{0} \mathrm{~d} y .
\end{aligned}
$$

At this point, by introducing

$$
h_{1}\left(x_{1}\right)=\mathrm{i} \frac{I_{2}\left(x_{1}\right)}{I_{1}\left(x_{1}\right)},
$$

eq. (31) can be rewritten as

$$
\mathrm{d}_{x_{1}} A\left(x_{1}\right)=\mathrm{i} h_{1}\left(x_{1}\right) A\left(x_{1}\right),
$$

which gives the following evolution of $A$

$$
A\left(x_{1}\right)=A_{0} \mathrm{e}^{\int h_{1}\left(x_{1}\right) \mathrm{d} x_{1}}=A_{0} \mathrm{e}^{\mathrm{i} H_{1}\left(x_{1}\right)},
$$

where $A_{0}$ is a constant of integration. Hence, up to a first order approximation,

$$
\varphi_{1}\left(x_{1}, y\right)=A\left(x_{1}\right) \zeta_{1}\left(x_{1}, y\right)
$$

and

$$
\psi=\left(\varphi_{0}+\varepsilon \varphi_{1}\right) \mathrm{e}^{\mathrm{i} \theta}=A\left(\zeta_{0}+\varepsilon \zeta_{1}\right) \mathrm{e}^{i \theta}=A_{0}\left(\zeta_{0}+\varepsilon \zeta_{1}\right) \mathrm{e}^{\mathrm{i}\left(\theta+H_{1}\right)} .
$$

From relations (7) and (8), the phase $\theta$ can be written as $\theta=h_{0} x-\sigma\left(x_{1}\right) t$, therefore

$$
\begin{aligned}
\theta+H_{1} & =h_{0} x+H_{1}\left(x_{1}\right)-\sigma\left(x_{1}\right) t \\
& =\int h_{0} \mathrm{~d} x+\int h_{1}\left(x_{1}\right) \mathrm{d} x_{1}-\sigma\left(x_{1}\right) t
\end{aligned}
$$




$$
=\int\left[h_{0}+\varepsilon h_{1}(\varepsilon x)\right] \mathrm{d} x-\sigma(\varepsilon x) t .
$$

Thus, $\varepsilon h_{1}$ represents the first order nonparallel correction to the complex wave number, which is now given by

$$
h\left(x_{1}, \sigma\right)=h_{0}+\varepsilon h_{1}\left(x_{1}, \sigma\right) .
$$

Eventually one obtains

$$
\psi=A_{0}\left(\zeta_{0}+\varepsilon \zeta_{1}\right) \mathrm{e}^{\mathrm{i}\left[\int\left(h_{0}+\varepsilon h_{1}\right) d x-\sigma t\right]} .
$$

It should be remarked that the loci of the stationary points of the dispersion relation (28), being an intrinsic property of the parametric Orr-Sommerfeld problem (21), cannot be influenced by the present procedure. The present perturbation approach only a posteriori determines corrected values of the perturbation complex wave numbers, which are now given by (32).

\section{The combined continuous spectrum for the parametric Orr-Sommerfeld problem}

In this section the continuous spectrum for the parametric Orr-Sommerfeld wake problem is considered within the context of the combined stability analysis. The aim is to verify if, in the light of Briggs criterium, all the physically acceptable countinuum modes are damped as found by Grosch and Salwen $[17,18,39]$ for both the temporal and the spatial stability problems of the parallel two-dimensional wake.

The search for improper eigenfunctions - eigenfunctions which do not have a finite norm - can lead to a continuous spectrum and can be carried out by relaxing the boundary conditions of eq. (21) to

$$
\begin{aligned}
\left|\varphi_{0}\right| & <\infty \text { as }|y| \rightarrow \infty, \\
\left|\partial_{y} \varphi_{0}\right| & <\infty \text { as }|y| \rightarrow \infty,
\end{aligned}
$$

as outlined by Grosch and Salwen [17,39]. In order to determine this kind of solution, it is useful to write eq. (21) in the asymptotic form

$$
\mathcal{A}_{\infty} \varphi_{0 \infty}=\sigma \mathcal{B} \varphi_{0 \infty}
$$

where

$$
\mathcal{A}_{\infty}=\left[\left(\partial_{y}^{2}-h_{0}^{2}\right)^{2}-\mathrm{i} h_{0} R\left(\partial_{y}^{2}-h_{0}^{2}\right)\right] .
$$

A fourth order equation with constant coefficients is thus obtained, which is independent of $x_{1}$. There are therefore four independent asymptotic solutions $\varphi_{0 \infty}(y)$ of the kind

$$
\left[\varphi_{0 \infty}\right]_{j}=\mathrm{e}^{\lambda_{j} y} \quad(j=1,2,3,4)
$$

with

$$
\begin{aligned}
& \lambda_{1,2}= \pm h_{0}, \\
& \lambda_{3,4}= \pm \sqrt{Q}= \pm \sqrt{h_{0}^{2}+\mathrm{i} R\left(h_{0}-\sigma\right)} .
\end{aligned}
$$

Eigenfunctions $\varphi_{0}$ of the complete eq. (21) will vary asymptotically like the linear combination

$$
\varphi_{0} \sim c_{1} \mathrm{e}^{\lambda_{1} y}+c_{2} \mathrm{e}^{\lambda_{2} y}+c_{3} \mathrm{e}^{\lambda_{3} y}+c_{4} \mathrm{e}^{\lambda_{4} y} \text { as }|y| \sim \infty
$$

In order to satisfy conditions (33) and (34), two of the four constants must be zero, as there is a corresponding diverging term for every vanishing term. Notice that, in such a way, also conditions (22) and (23) for proper eigenfunctions are satisfied. On the other hand, it is possible to satisfy conditions (33) and (34) for improper eigenfunctions without satisfying the conditions (22) and (23), which can instead be done when the coefficients $\lambda_{j}$ are purely imaginary. In this case, condition (36) becomes:

$$
\Re\left\{h_{0}\right\}=k_{0}=0
$$

and condition (37) becomes:

$$
\Re\left\{(Q)^{\frac{1}{2}}\right\}=0 .
$$


Condition (38), also when $s_{0}=0$, leads to a degeneration of eq. (21), so that only the trivial eigenfunction $\varphi_{0}=0$, with eigenvalue $\sigma=0$, is obtained. When $s_{0} \neq 0$, operators $\mathcal{A}_{\infty}$ (35) and $\mathcal{B}$ become real, the eigenvalue $\sigma$ becomes imaginary (standing waves) and can be included in the set of eigenvalues which satisfy condition (39).

Condition (39) is equivalent to

$$
\begin{aligned}
& \Re\{Q\}<0 \Longrightarrow k_{0}^{2}-s_{0}^{2}+R\left(r-s_{0}\right)<0, \\
& \Im\{Q\}=0 \Longrightarrow 2 k_{0} s_{0}+R\left(k_{0}-\omega\right)=0 .
\end{aligned}
$$

These relations can be rewritten as

$$
\begin{aligned}
\omega & =\frac{k_{0}}{R}\left(R+2 s_{0}\right), \\
r & =\frac{1}{R}\left[s_{0}\left(R+s_{0}\right)-k_{0}^{2}-\nu\right],
\end{aligned}
$$

where a real parameter $\nu>0$ has been introduced to satisfy the inequality (40). It can be remarked that, among all the possible complex values of the wave number, which is the parameter in the condition (39), the imaginary ones $\left(h_{0}= \pm \mathrm{i} s_{0}\right)$ can also satisfy conditions (38). Condition (39) in turn yields eigenvalues, $\omega=0$ and $r=(1 / R)\left[s_{0}\left(R+s_{0}\right)-\nu\right]$, which are purely imaginary. Thus, according to the conbined analysis, the four branches (Grosch and Salwen [18,39] of the continuous spectrum are given by $\sigma_{\nu_{1,2}}= \pm \mathrm{i}(1 / R)\left[s_{0}\left(R+s_{0}\right)-\nu\right]$ and by $\sigma_{\nu_{3,4}}= \pm(1 / R)\left[k_{0}\left(R+2 s_{0}\right)+\mathrm{i}\left(s_{0}\left(R+s_{0}\right)-k_{0}^{2}-\nu\right)\right]$.

The search for branching points in the continuous spectrum can now be carried out analytically. Given that

$$
\sigma_{\nu}\left(h_{0}, R\right)=\frac{1}{R}\left\{R h_{0}-\mathrm{i} h_{0}^{2}-\mathrm{i} \nu\right\}(\nu>0),
$$

by calculating $\partial \sigma / \partial h_{0}$, one obtains

$$
\frac{\partial \sigma}{\partial h_{0}}=1-\mathrm{i} \frac{2}{R} h_{0},
$$

so that the Briggs condition (29) yields $h_{0}=-\mathrm{i}(R / 2)$ and $\sigma_{\nu}=-\mathrm{i}(\nu+R / 4)$. These relationships represent strongly damped standing waves with a phase speed $\Re\{c\}=r / s_{0}=1 / 2+2 \nu R$, where $c=h_{0}^{*} \sigma /\left|h_{0}\right|^{2}$ is the combined complex phase speed.

Note that, since the asymptotic form of the parametric Orr-Sommerfeld equation coincides with that of the parallel case, this result, as well as the eigenvalues continuous spectrum, is independent on $x_{1}$. Thus, according to the near parallel assumption, a dependence on $x_{1}$ is recovered at the level of only the eigenfuntions and for only finite values of $y$. The present continuous spectrum scenario, which was obtained by means of both the combined stability analysis and the Briggs criterium, does suggest a remarkable stable - given that the minimum damping ratio of single modes is equal to $-R / 4-$ behaviour of the corresponding perturbation wave packet. As a consequence, the determination of the first order nonparallel corrections $(O(\varepsilon), \varepsilon=1 / R)$ to the countinuum spatial stability characteristics, as here obtained, does not seem necessary.

\section{Nonparallel correction for the local linear stability analysis of the cylinder wake}

Since the basic flow varies along the streamwise direction, the dispersion relation becomes a function of coordinate $x$. In this section the discrete eigenvalue spectra corresponding to several $x$ stations in the intermediate and far flow regions have been analyzed. The assumptions that the primary cylinder wake is slightly nonparallel and can be considered slowly varying with $x$ made it possible for the method of multiple, spatial, scales to be adopted (see Sects. 1 and 2). However, before discussing these results, it is interesting to consider which streamwise variations the complex wave number $h$ undergoes, as the after-body is approached from infinity downstream. The spatial deformation at $R=50$ of a rectangular portion $P$ of the complex wave number plane, $\left(k_{0} \in[0.4,0.8] ; s_{0} \in[-1.5,-0.5]\right)$ is shown in Fig. 1. In part (a) of this figure, $x=100$ and the first order corrections are neglegible, so that $P$ is not deformed. It can be remarked that this picture also represents the zero order complex interval $P$ at any value of the coordinate $x$. In part (b), $x=20$ and the variations of both the wave number, $\varepsilon k_{1}$, and the spatial growth rate, $\varepsilon s_{1}$, have become remarkable. In part (c), $x=10$ and it is evident that the first order corrections are certainly not small corrections, in fact the variations reach maximum values of about $100 \%$. The multiscale approach and the locally parallel approximation are no longer valid here. Getting closer to the body, part (d), $x=5$ and the first order corrections are larger than the zero order values. Multi-valued points appear in the transformed portion $P$. It should be recalled that $x=5$ (at $R=50$ ) is approximatively the end of the two cylinder standing eddies, which length, according to a by now very established body of experimental and numerical results (see e.g. Nishioka and Sato [33]; Fornberg [14] and the review by Coutancaeau and Defaye [11]), is of about 5 body scales. From this result, which 

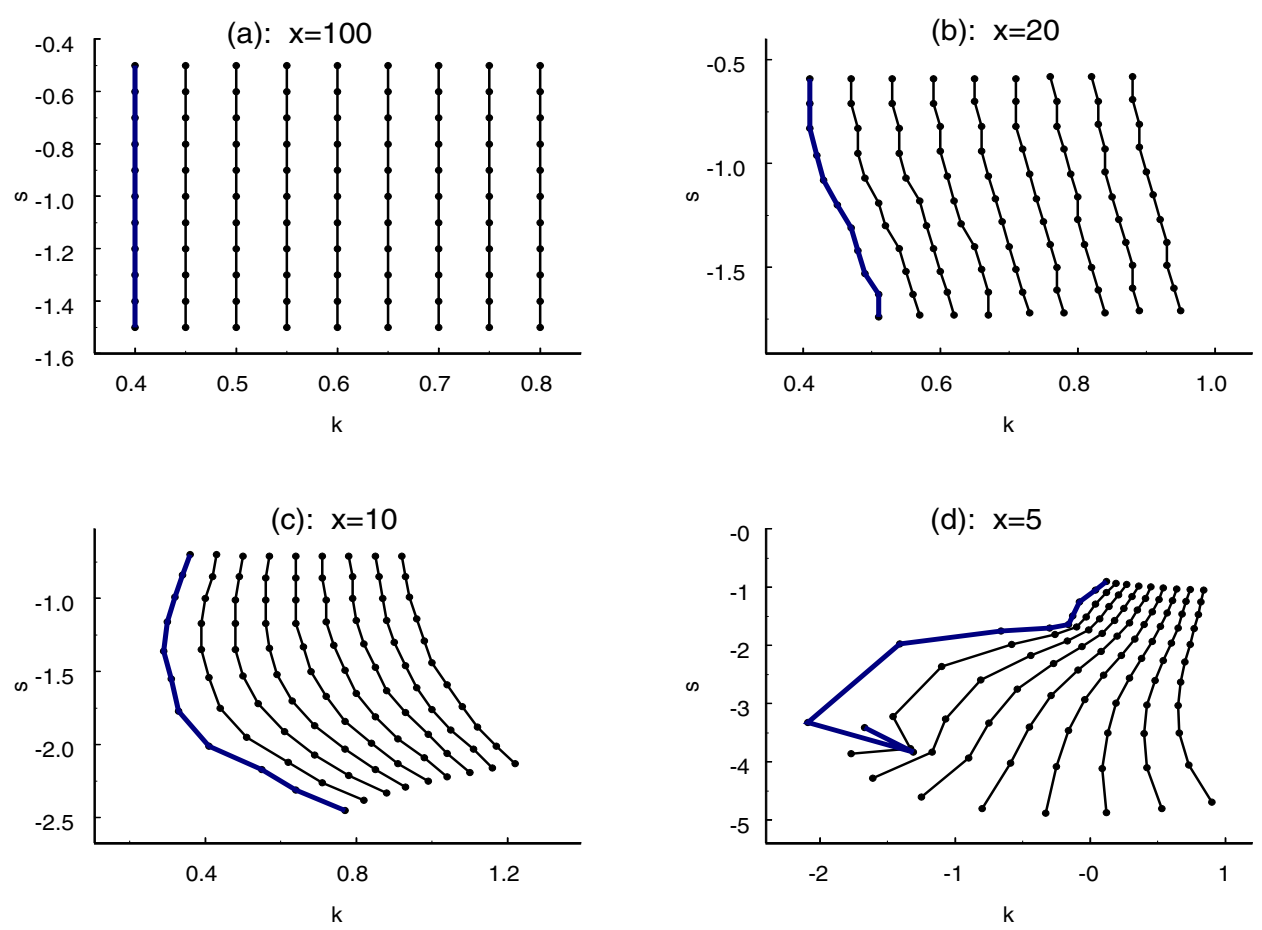

Fig. 1 Deformation of a rectangular portion of the complex wave number domain at $R=50$; $\mathbf{a} x=100$, b $x=20$, c $x=10, \mathbf{d} x=5$.
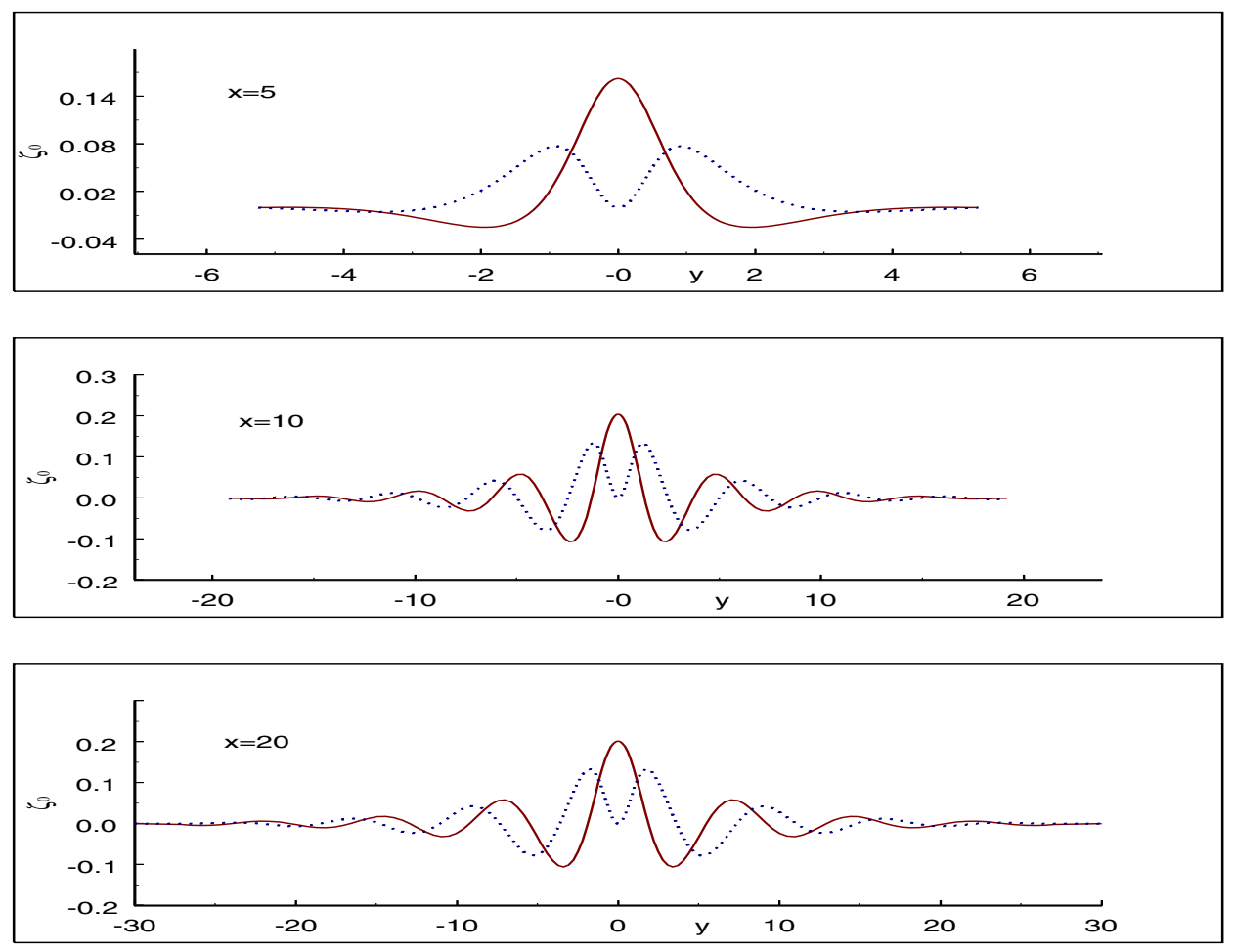

Fig. 2 Profiles of the perturbation stream function $\zeta_{0}$ at different streamwise stations, - real part, .... imaginary part. 


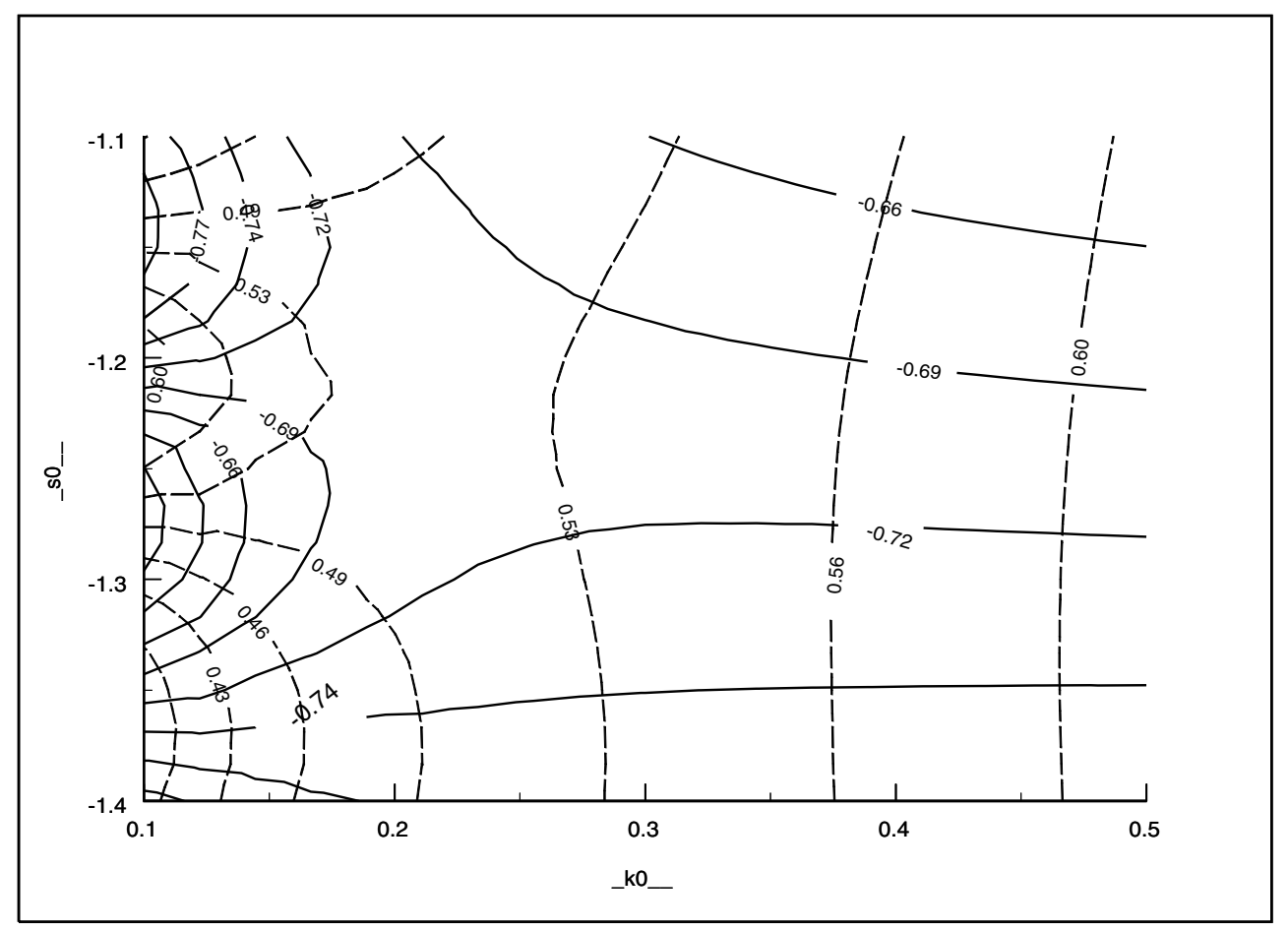

Fig. $3 R=50, x=10$, map of frequencies - - - and temporal growth rates — in the complex wave number plane about the branching point $h_{0}=0.23-1.21 i, \sigma=0.52-0.72 i$.

has been confirmed for all the $R$ numbers considered in this study ( $R \in[30,100])$, the inference can be drawn, that the locally parallel flow assumption loses significance well outside the standing vortices.

The variation of the eigenvalue spectra along $x$, and in particular the variation of the stationary points and relevant branching points, as described in Sects. 2.2 and 2.3, it is now considered. First, let us present the plot of the perturbation stream function $\zeta_{0}$ profiles at different streamwise stations in Fig. 2, which shows an $x$ increasing lateral spreading, and, as a typical sample, the map (at $x=10$ and $R=50$ ) of the frequencies and temporal growth rates about the saddle point $k_{0}=0.52, s_{0}=72$ in Fig. 3. Since Betchov and Criminale [9] first discovered them, the existence of wake branchpoint singularities has been determined by many authors using the local parallelism assumption, see e.g. Mattingly and Criminale [26], Koch [24], Hultgren and Aggarwal [21], Monkewitz [27], Yang and Zebib [52], Hannemann and Oertel [19]. As a consequence, quite a number of $O\left(\varepsilon^{0}\right)$ approximations of the corresponding loci exist and can be used, as first guess values, to start the search for the saddle points in the wave number plane, which was here carried out according to Deissler's procedure [12]. The important new information that the present analysis gives is gathered in the sequence of Figs. 4-6, which show, at $R=35,50,100$, apart from the frequency and temporal growth rate distributions of the branching points, the $x$ dependence of the real and imaginary parts of $h$, both at the zero and first order of accuracy with regards to $\varepsilon$. The main finding is that the first order corrections that characterize the real and imaginary part of $h_{0}$ becomes already very large in the intermediate wake well downstream to what is usually considered the near wake, i.e. the portion of flow just behind the body, where the standing vortices are found and the local parallel analysis finds sub-regions of absolute instability. This is partly an unexpected result. While, on one hand, a failing of the near-parallelism assumption in the near wake, which is the region of the wake where the curvature of the streamlines is maximum, was obviously expected, on the other hand it was expected that the corrections could still be considered corrections - so that

$$
\left|\frac{1}{k} \frac{\partial(k)}{\partial x}\right| \ll \frac{1}{\lambda(x)}\left(\sim \frac{1}{x}, \text { as } x \rightarrow \infty\right),
$$

is verified - in most of the intermediate wake. In other words, the progressive deterioration of the validity of the local parallel assumption was presumed to result in a break up near the region where the temporal growth rates become positive. Such a result could have been effective in supporting the assumption under discussion, since, by extrapolation from the intermediate wake, approximated information could be drawn for the instability characteristics inside the body scale region of the flow (see Fig. 7). 

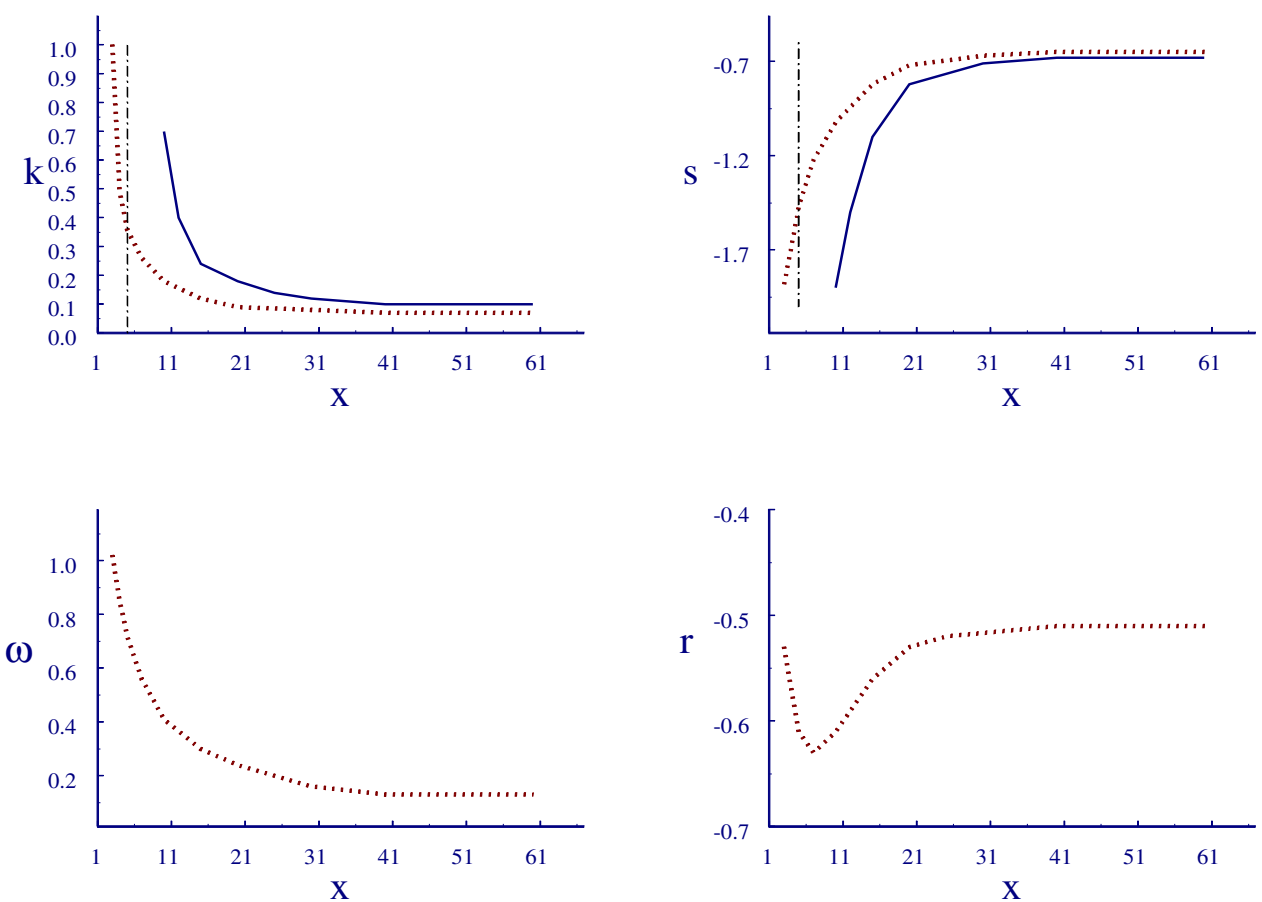

Fig. 4 Streamwise distribution of the instability characteristics at the stationary points, $R=35 ; \ldots . . O\left(\varepsilon^{0}\right),-O(\varepsilon)$, -.-.- length of the standing eddies.
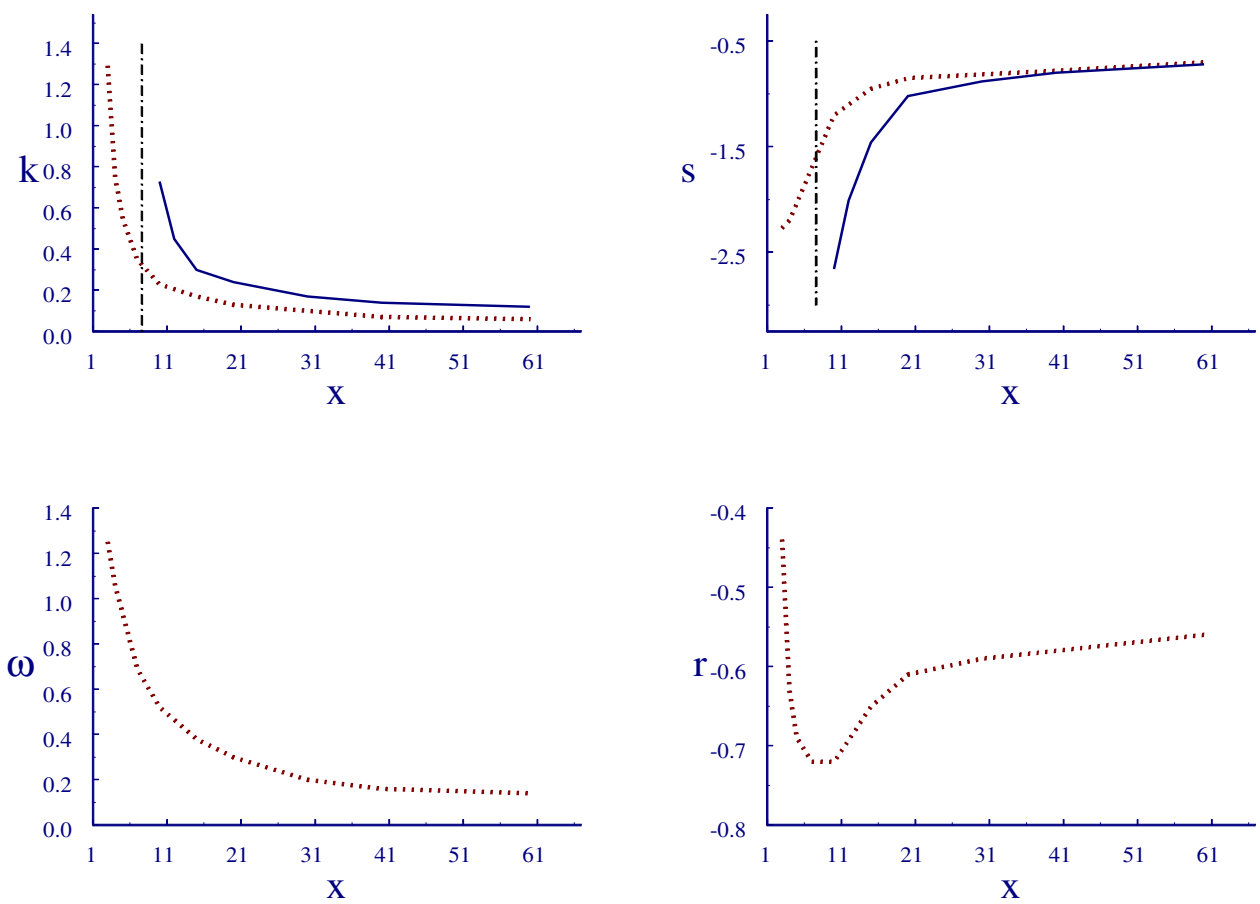

Fig. 5 Streamwise distribution of the instability characteristics at the stationary points, $R=50, \ldots . . O\left(\varepsilon^{0}\right),-O(\varepsilon)$, -.-.- length of the standing eddies.

In such a situation, it can be concluded (for $R \in[35,100]$ ) that:

i) the scaling for the correction of nonparallelism must be based on the steady length scale $l$, which is generated by the dynamics of the flow, and which is equal to the sum of the body scale, the diameter $D$ of the cylinder, and the length $L_{e} \sim R$ (i.e. $l=1+L_{e} / D$ ), where $L_{e}$ is the scale of the symmetric standing eddies attached to the body (which, at $R=100$, is already one order of magnitude greater than $D$ ), 

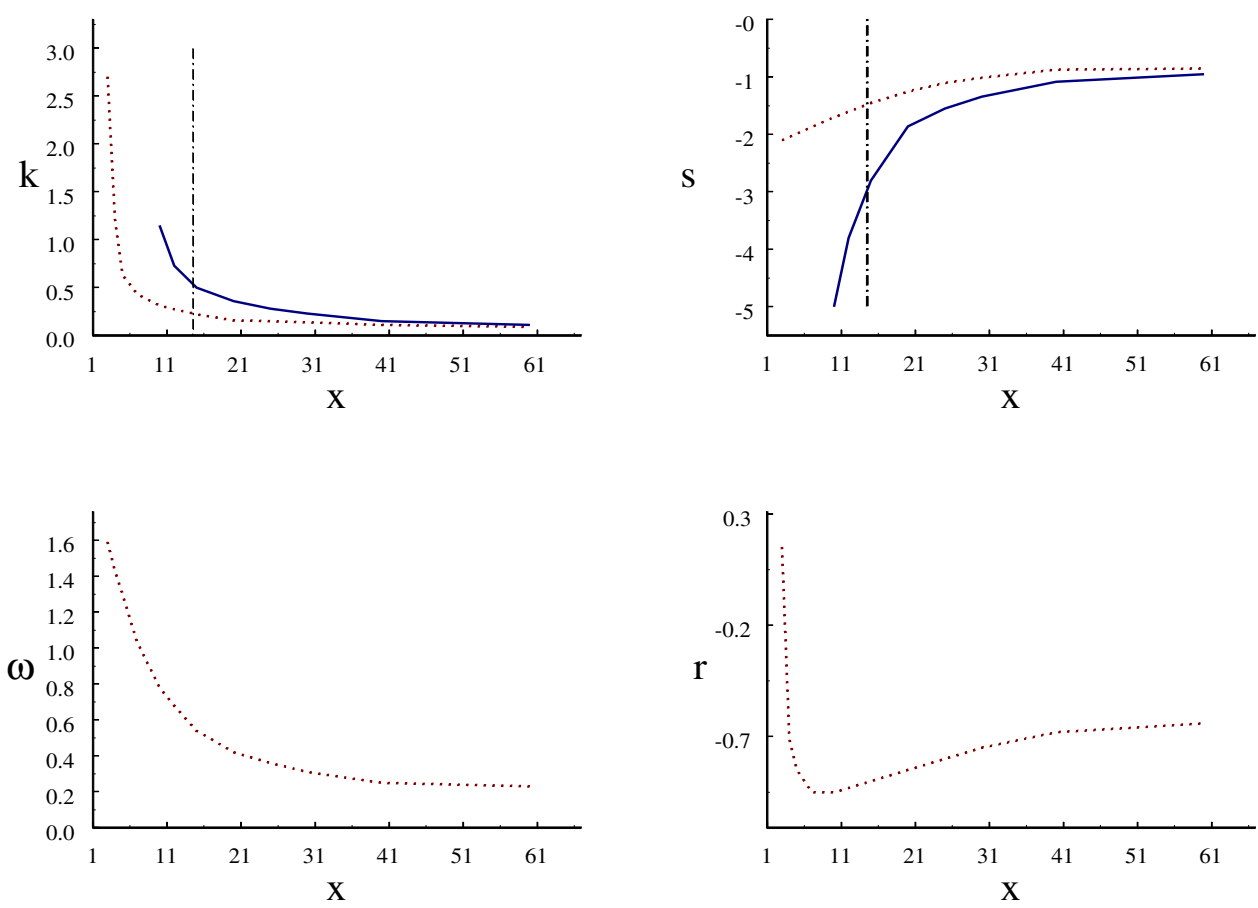

Fig. 6 Streamwise distribution of the instability characteristics at the stationary points, $R=100, \ldots . . O\left(\varepsilon^{0}\right),-O(\varepsilon)$, -.-.- length of the standing eddies.

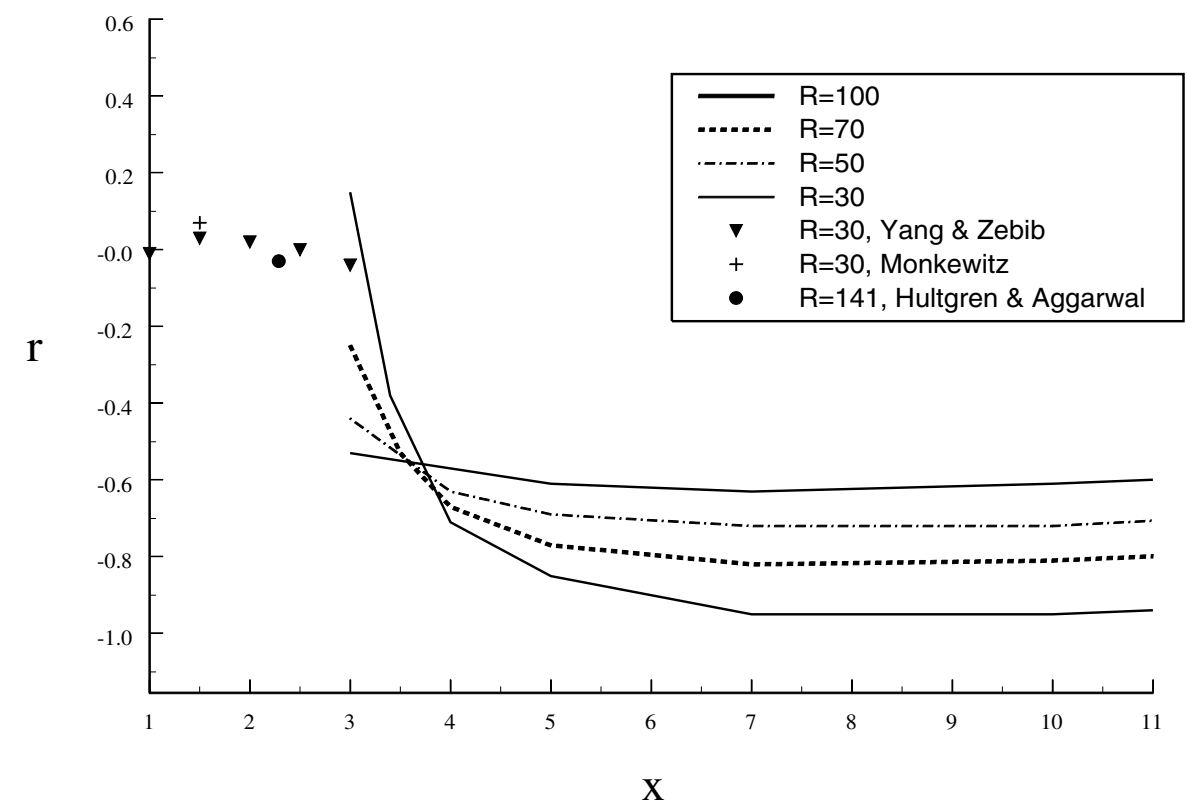

Fig. 7 Streamwise distribution of the temporal growth factors at the branching points of the local parallel flow dispersion relation of the circular cylinder wake at different Reynolds numbers. Comparison with calculations [21] •, [27] +, [52]

ii) the hypothesis of near parallelism starts to be self-consistent from $2 \approx 5 l$ scales downstream to the body, depending on the Reynolds number ( $5 l$ at $R=35,2 l$ at $R=100$ ). As a consequence, in the near wake, the values of the spatial stability characteristics forecast by the near-parallelism assumption must be used with caution. Beyond about 3 to $10 l(x>10 l$ at $R=35, x>6 l$ at $R=50, x>3 l$ at $R=100$ ) the zero and first order theories coincide.

A summarising plot of the streamwise variation of the temporal growth rates is given in Fig. 7, with $R$ as the parameter, for comparison with previous local stability analysis results. 


\section{Conclusions}

An analysis of the influence of the flow nonparallelism over the stability properties produced by the local parallel theory, as applied to the two dimensional incompressible bluff body wake, has been presented. The nonparallel corrections to the propagation and growth characteristics of combined normal modes that are relevant to a primary flow, which is described by an asymptotic Navier-Stokes expansion solution and which is valid in the intermediate and far wake, were determined using a multiscale spatial approach. The quasi-parallel assumption gives results which coincide with the results obtained from the nonparallel correction in the region of the flow beyond 3 to 10 , depending on the Reynolds number, steady flow scales $l$ downstream to the body (where $l=1+L_{e}(R) / D$, and $L_{e}$ is the standing eddies length, $R \in[30,100]$ ). Getting closer to the body, the spatial instability characteristics depend to a great extent on the nonparallelism of the basic flow. Both the wave number and the spatial growth rate corrections increase while approaching the body from downstream, until, well before meeting the standing eddies wake, the break up of the perturbation approach occurs. The instability scenario here obtained is the following. In the intermediate and far regions, at order $\left(\varepsilon^{0}\right)$, the instability results to be convective. At this order, as almost all the former quasi-parallel instability studies have discovered, a zone of absolute instability could be envisaged just after the body, inside the standing eddies, by extrapolating the trends of the instability characteristics in the intermediate zone. Nevertheless, this result is obtained through the local parallel flow analysis exactly in the region were the assumption on which it relies is not valid, as the nonparallel correction behaviour obtained here demonstrates.

The continuous spectrum of the parametric Orr-Sommerfeld problem has also been considered within the context of the combined stability analysis. According to the Briggs criterium, it is shown that the continuum single modes are stable.

A theoretical inference is that the extension of the present multiscale normal mode instability approach to a superior order of accuracy, $O\left(\epsilon^{2}\right)$, would be an unjustified effort.

\section{References}

[1] M. Abramowitz and I.A. Stegun, Handbook of Mathematical Functions (Dover, New York, 1965).

[2] G. I. Barenblatt, Scaling, Self-similarity, and Intermediate Asymptotics (Cambridge University Press, Cambridge, UK, 1996), Preface, p. XIII.

[3] M. Belan and D. Tordella, Z. Angew. Math. Mech. 82 4, 219-234 (2002).

[4] E. Berger, Phys. Fluids 10(Suppl.), 191-193 (1967).

[5] E. Berger, R. Wille, Annu. Rev. Fluid Mech. 4, 213-240 (1972).

[6] S. Berrone, Adaptive Discretizations of Differential Models in Fluid Dynamics, Ph.D. Dissertation, Politecnico di Torino (2001).

[7] S. Berrone, Comput. Methods Appl. Mech. Eng. (Netherlands) 190(34), 4435-4455 (2001).

[8] A. Bers, in: Physique des Plasmas, edited by C. De Witt and J. Peyraud (Gordon and Breach, London, 1975), pp. 117-213.

[9] R. Betchov and W. O. Criminale, Phys. Fluids 9(2), 359-362 (1966).

[10] R. J. Briggs, Electron Stream Interaction with Plasmas, Research Monographe 29 (MIT Press, Cambridge, USA, 1964).

[11] M. Coutanceau and J. R. Defaye, Appl. Mech. Rev. 44(6), 255-281 (1991).

[12] R. J. Deissler, Phys. Fluids 30, 2303-2305 (1987).

[13] S. C. R. Dennis and G.Z. Chang, J. Fluid Mech. 42(3), 471-489 (1970).

[14] B. Fornberg, J. Fluid Mech. 98, 819 (1980).

[15] M. Gaster, J. Fluid Mech. 38, 565-576 (1969).

[16] M. Gaster, J. Fluid Mech. 46, 749-756 (1971).

[17] C.E. Grosch and H. Salwen, J. Fluid Mech. 87, 33-54 (1978).

[18] C. E. Grosch and H. Salwen, Bull. Am. Phys. Soc. 23, 525 (1978).

[19] K. Hannemann and H. Oertel, J. Fluid Mech. 199, 55-88 (1989).

[20] P. Huerre and P. A. Monkewitz, Annu. Rev. Fluid Mech. 22, 473-537 (1990).

[21] L. S. Hultgren and A. K. Aggarwal, Phys. Fluids 30(11), 3383-3387 (1987).

[22] C. P. Jackson, J. Fluid Mech. 182, 23-45 (1987).

[23] E. Kamke, Differentialgleichungen (B. G. Teubner, Stuttgart, 1977).

[24] W. Koch, J. Sound Vib. 99(1), 53-83 (1985).

[25] L. S. G. Kovásznay, Proc. R. Soc. Lond. A 198, 174-190 (1948).

[26] G. E. Mattingly and W. O. Criminale, J. Fluid Mech. 51, 233 (1972).

[27] P.A. Monkewitz, Phys. Fluids 31(5), 999-1006 (1988).

[28] P.A. Monkewitz and L. N. Nguyen, J. Fluids Struct. 1, 165-184 (1987).

[29] M. Morzinsky and F. Thiele, Z. Angew. Math. Mech. 71, T424-T428 (1991).

[30] M. Morzinsky and F. Thiele, Z. Angew. Math. Mech. 72, T403-T407 (1992).

[31] A. H. Nayfeh, Perturbation Methods (Wiley, New York, 1973).

[32] B. R. Noak and H. Eckelmann, J. Fluid Mech. 270, 297-330 (1994).

[33] M. Nishioka and H. Sato, J. Fluid Mech. 65(1), 97-112 (1974).

[34] H. Oertel, Annu. Rev. Fluid Mech. 22, 530-546 (1990).

[35] R.T. Pierrehumbert, J. Atmos. Sci. 41, 2141-2162 (1984). 
[36] M. Provansal, C. Mathis, and L. Boyer, J. Fluid Mech. 182, 1-22 (1987).

[37] A. Roshko, J. Aerosol Sci. 22, 124-132 (1955).

[38] M. Roussopoulos, J. Fluid Mech. 248, 267-296 (1993).

[39] H. Salwen and C.E. Grosch, J. Fluid Mech. 104, 445-465 (1981).

[40] W. S. Saric and A. H. Nayfeh, Phys. Fluids 18(8), 945-950 (1975).

[41] P. J. Strykowski and K. R. Sreenivasan, J. Fluid Mech. 218, 71-107 (1990).

[42] P. Sturrock, Phys. Rev. 112, 1488-1503 (1958).

[43] D. Tordella and M. Belan, Phys. Fluids 15 (7), 1897-1906 (2003).

[44] G. S. Triantafyllou, K. Kupfer, and A. Bers, Phys. Rev. Lett. 59(17), 1914-1917 (1987).

[45] G. S. Triantafyllou, M. S. Triantafyllou, and C. Chryssostomidis, J. Fluid Mech. 170, 461-477 (1986).

[46] G. S. Triantafyllou and G. E. Karniadakis, Phys. Fluids 2 (5), $653-656$ (1990).

[47] D. J. Tritton, J. Fluid Mech. 6, 547-567 (1959).

[48] D. J. Tritton, J. Fluid Mech. 45, 203-208 (1971).

[49] R. Q. Twiss, Phys. Rev. 88, 1392-1407 (1952).

[50] G. B. Whitham, Linear and nonlinear waves (John Wiley and Sons, Chichester, Sussex, UK, 1974).

[51] C. H. K. Williamson, Phys. Fluids A 31, 2742-2744 (1988).

[52] X. Yang and A. Zebib, Phys. Fluids A 1(4), 689-696 (1989).

[53] A. Zebib, J. Eng. Math. 21, 155-165 (1987). 